\title{
LA DICOTOMFA ESTRUCTURAL EN LA SOCIEDAD LATINOAMERICANA
}

\author{
Carlos M. Rama \\ (Universitat Autònoma de Barcelona)
}

$\mathrm{EI}$ análisis de las estructuras sociales dependientes en la era capitalista definidas históricamente por una dicotomía estructural es un tema clásico en la moderna investigación. El presente ensayo ofrece un recorrido analítico por las diversas orientaciones más relevantes que se han ocupado del tema, resaltando al mismo tiempo la validez científica como las ambigüedades de sus aportaciones y confrontaciones polémicas. 
El tema de la dicotomía ${ }^{3}$ estructural de las sociedades de la época capitalista ya había sido señalado por los economistas clásicos de la lengua inglesa en el siglo xvin, y lo recogió Catlos Marx en El Capital, cuando destaca que: «La base de todo régimen de división del trabajo, un poco desarrollado y condicionado por el intercambio de metcadetias, es la separación entre la ciudad y el campo. Puede decirse que toda la historia económica de la sociedad se tesume en la dinámica de este antagonismo, en cuyo análisis no podemos detenernos aquí.» ${ }^{2}$

1. El origen y definición gtamatical de esta palabra lo muestra el Diccionario de la Lengua española (Madrid: Real Academia Española, 1970), 19a. edición, en su aceptación lógica, como «Método de clasificación en que las divisiones y subdivisiones sólo tienen dos partes». En cuanto a la palabra dualismo (puede considerarse sinónima) se define como «Condición de reunir dos caracteres distintos en una misma persona - cosa». Ni el Lalande, Vocabulaire tecbnique et critique de la pbilosopbie (París: Presses Universitaires đe France, 1970), 9a. edición, ni el Diccionario de Sociologia de Fenry Pratt Faitchild (México: Fondo de Cultura Econónica, 1949), 1a. edición, definen estos términos. José Ferrater Mora en su Diccionario de filosofía (Buenos Aires: Sudamericana, 1958), 4a. edición, p. 358, define dicotomía como «la división de un concepto en dos conceptos contrarios que agotan la extensión del primero» y en Ia p. 385 , se refiere a la introducción de dualismo en la historia de la fitosofía como proveniente de la historia religiosa, observando que fue Thomas Hyde quien en su Historia religiosa de los persas para referirse al maniqueísmo persa religioso empleó por vez primera aquel térnino, que actualmente significa «toda contraposición de dos tendencias irreductibles entre sír. La definición que hemos establecido para América Latina es la siguiente: «Concepto explicativo sobre las sociedades tatinoamericanas que destaca la existencia, dentro de cada una de las sociedades globales (un país, en la terminología, por ejernplo, de George Gurvitch), de dos sociedades situadas a muy distintos niveles de evolución, por una parte una sociedad rural atrasada y por otra una sociedad urbana en que se destacan acusadamente rasgos de modernidad. La coexistencia de ambas sociedades implica tensiones, luchas y dualismos en los niveles económicos, sociales y políticos, y hasta históricos. También supone una distinta relación con el mundo exterior, representado normalmente por sociedades industrializadas de más alto desarrollo,» Véase: Diccionario de Ciencias Sociales (Madrid, 1974), Vol. I.

2. Vol. I, cap. XIX, p. 286, según cuarta reimpresión en edición de México: 
Entre los primeros autores latinoamericanos que cultivaron en el siglo XIX las ciencias sociales tampoco failtó la consideración de este tema, y en ocasiones - como en el caso del argentino Domingo Faustino Sarmiento- con su famoso Facundo Quiroga. Civilización y barbarie (1845), hizo del punto la base de todo un original sistema sociológico interpretativo.

Entre la ciudad herramienta de civilización y la campaña donde predominaba hegemónicamente la barbarie se establecería una relación histórica de tal importancia que podría servir de teoría explicativa de todo el proceso político, social y cultural ameticano. ¿Cuáles fueron las raíces del pensamiento sarmentiano? En general, se ha intentado rastrear su filiación en el pensamiento social presociológico francés como el de Montesquieu, o en la historia crítica como en Alexis de Tocqueville.

Naturalmente esa corriente ve en las ciudades especialmente su carácter genezador de cultura, desarrollo econónico y progreso político. Hay una segunda corriente que considera a la ciudad como parasitaria de Ias riquezas creadas por el campo (que estaba ya presente en las críticas de Juan Bautista Alberdi a su contemporáneo Sarmiento), pero en el fondo ambas coinciden en reconocer dos sociedades $y$, por tanto, dos econonías que dualizan el cuadro nacional de cada país latinoamericano. ${ }^{3}$

Éstos son antecedentes calificados de las sugerencias que resultan de la obra del profesor Jacques Lambert sobre el Brasil, donde se desarrolla prolijamente la tesis de los «dos Brasil», que apunta a este tema. Señala concretamente Lambert que: «Los brasileños están divididos en dos sociedades, por diferentes niveles y modos de vida, de las cuales una es más especiffcamente rutal y la otta más especificamente urbana; ambas han evolucionado a distinto ritmo y no han llegado al mismo estadio; no están separadas por una diferencia de naturaleza, sino por diferencias de edad. ${ }^{4}$

Aunqute este texto hace referencia a niveles $y$ modos de vida, y ade-

Fondo de Cultura Económica, 1971. Marx cita en nota al pie la obra de los economistas James Stewatt (1767) y Adam Smith (1814) como sus fuentes.

3. Los argumentos de Alberdi son: que la sociedad latinoameticana independiente no era distinta de la sociedad colonial, y «Las Leyes de Indias tuvieron desterradas de estas ciudades, las ciencias, las artes, las fábricas, las máquinas, los oficios liberales, las industrias y las letras mismas», en tanto las campiñas «representan la barbarie, porque encierran toda la riqueza, todo el ser, todo el valor y la vitalidad del paísn. Textos sacados de los artículos «Palabras de un ausente en que explica a sus amigos del Plata los motivos de su alejamienton y «La Civilización moderna es la seguridad» incluidos en el volumen de Víctor Rico González (ed.), Iuan Bautista Alberdi (México: Universitaria, 1946), pp. 115-151.

4. Jacques Lambert, Le Brésil. Structures sociales et institutions politiques ( $\mathrm{Pa}$ rís: A. Colin, 1953), pp. 64-82. 
más los hace coincidir grosso modo con sociedad rural y sociedad urbana, más adelante considera la dicotomía como expresión de diferentes culturas (culture brésilienne archaique, etc.); también recurre a la explicación dinámica (la nueva sociedad «mucho más evolucionada y mucho más móvil»), e incluso parece apuntar al tema de las relaciones sociales porque la sociedad rural sería aislada, y «lleva todavía la marca del siglo xvi», y aún -final. mente- determinada por la supervivencia o consecuencia de ciertas instituciones, porque siempre la sociedad atcaica muestra la presencia de la esclavitud.

El Brasil rural entonces engloba la mayoría de la población del país, o sea de un 60 a un $70 \%$ del total nacional, incluyendo grupos urbanos, del mismo modo que el Brasil urbano o adelantado, gira alrededor de São Paulo «y de algunas otras regiones al sur del país». Se caracteriza por «una estructura social menos jerarquizada, mucho más compleja: una estructura que cambia tápidamente». Y concluye: «Entre esas dos edades en la cuitura brasileña la herida no ha cesado de acentuarse, puesto que una ha evolucionado más y más rápidamente, mientras que la otra permanece perfectamente inmóvil hasta el punto de que su aislamiento no se ha roto.»

Este conjunto de ideas, o mejor de sugerencias, están incluidas en la segunda parte del Capítulo III \& «a structura taciale et la structure sociale») de Le Brésil. Structure sociale et institutions politiques (1953), en que el profesor de Lyon recoge el fruto de una larga estadía de estudios en Brasil, donde ejerciera la cátedra de Sociología de la Universidad Federal entre 1939 y 1945, como miembro de la Misión de Profesores Franceses.

Tan importantes fueron consideradas esas ideas, que cuando se tra. dujo el libro al portugués editándose por el Centro Brasileiro da Pesquisas Educacionais del Ministerio da Educaçäo e Cultura de Río de Janeiro en el año 1959, la obra pasó a llamarse Os dois Brasis, ${ }^{5}$ y el Capitulo III antes citado se dividió en dos capítulos, de los cuales el ahora quinto tomó et nombre de «A sociedade dualista e o contraste de estructura social entre os dois Brasis» (pp. 105-126). Las conclusiones son las mismas que en 1953, aunque no faltan correcciones de detalle. Todo esto corresponde precisarlo para destacar cómo las tesis de Jacques Lambert fueron puestas, en implícito acuerdo y apoyo, en conocimiento del público lector brasileño.

Lambert vio en su tesitura una regla de aplicación general para otros

5. Se trataba del primet volumen de la serie «Sociedade e Educação» que dirigía Darcy Ribeiro; Jacques Lambert en un breve prefacio le agradece junto a Anixio Teixeira, Alceu Amoroso Dima, L. A. Costa Pinto, José Honorio Rodríguez y otros destacados brasileños que pocos años más tarde se contarán como figuras centrales del stoff intelectual del intento populista del gobierno de João Govlatt hasta 1964. 
países sudameticanos, aparte de Brasil, en los cuales la civilización industrial ha sido brutalmente importada (son sus palabras) por contraste con Europa o los nuevos países anglosajones, donde la civilización industrial se ha desarrollado progresivamente. ${ }^{6}$ En otra parte, sin embargo, parece exceptuar (dentro siempre de América del Sur) del dualismo o dicotomía social estructural a las vecinas repúblicas de Argentina y Uruguay.

Para México ha sido Pablo González Casanova quien ha aplicado en fechas recientes los conceptos de dicotomía, con expresa mención de la obra de Jacques Lambert sobre Brasil. Con el título «El México que tiene y el que no tiene», publicó un texto en una conocida revista mexicana, que después integró en 1965 como capítulo de su libro La democracia en México. ${ }^{7}$

Según este autor, «una de las características más generales de los países subdesarrollados es la estructura dual o plural de sus sociedades, la heterogeneidad cultural, económica, política, que divide a cada país subdesarrollado en dos o más mundos de tal modo distintos, que el investigador se ve impulsado a hablar de dos o más países, como Lambert, que escribe su libro sobre Os dois Brasis, o Simpson, que escribe el suyo intitulado Many Mexicos». Se aprecia en el texto que los «dos países mexicanos» son el rural y el urbano, y esto se afirma en el aparato estadístico correspondiente.

De la explicación dualista tenemos el caso que considera el conjunto de América Latina, pero con una visión de tipo geográfico general; un ejemplo lo encontramos en un texto del profesor francés Frédéric Mauro. Éste, bajo la denominación de "Amérique Latine Orientale et Amérique Latine occidentale: une apposition historique», ${ }^{8}$ llama la atención «sobre una oposición que hasta ahora no ha sido descrita -por lo menos, según

6. Lambert se manejaba con un cuadro de referencias directas no solamente sobre Francia, también sobre los Estados Unidos, país sobre el cual había escrito la obra en colaboración Les fondements de la politique extérieure des Etats Unis (París, A. Colin). Su conocimiento, en cambio, del resto de América es insuficiente, aunque reconoce con exactitud que Árgentina y Uruguay tienen características que les distinguen, y en especial, presentan una introducción antigua de la industrialización, que abrevia su estadio colonial (véase J. Lambert, Le Brésil..., op. cit., pp. 9 y 83).

7. Siempre, núm. 526 (suplemento «La cultura en México»). La citada obra fue editada por ERA, y tiene a la fecha numerosas reediciones. El texto citado también se incluye en el volumen ¿Ha muerto la revolución mexicana? Balance y epilogo. (México: Sep-Setentas, 1972.) Vol II, traducción de la vetsión inglesa (Nueva York: Alfred A. Knopf, 1966).

8. Véase el volumen Études offertes à Jacques Lambert (París: Editions Cujas, 1975), pp. 53-64. 
nuestro conocimiento- y que, sin embargo, puede orientar en parte, en el porvenir, el destino del hemisferio occidental; la que existe entre la América Latina "oriental" y la América Latina "occidental" [...] ponemos en la primera las Antillas, Venezuela, Guayana, el conjunto brasileño y los Estados del Río de la Plata. En la América occidental colocamos los Estados andinos y de los de América Central continental».

Este planteo se reclama asimismo de Lambert, pero aparentemente es exagerado, porque si bien es cierto que existe una diferenciación geográfica, histórica, cultural, etc., no alcanza el grado de un dualismo dicotómico, que es el concepto implícito en las demás obras que tratan del tema. Oponer Caracas a Lima, o la población rural brasileña a la chilena, no parece explicativo de los problemas del subcontinente.

Las opiniones o teorías dicotómicas han sido contestadas o rechazadas, y se deben anotar ciertas menciones de interés.

A) En lo que hace referencia a Europa, cuando en el año 1966 el historiador Arnold J. Toynbee visita Brasil, reencuentra la hipótesis del dualismo como explicativo de ciertos fenómenos sociales. «En el Brasil -dice- hay dos naciones con dos modos de vida muy diferentes.» Pero agrega, rechazando implícitamente la idea de Lambert según la cual ese fenómeno es desconocido en Europa: «Será tan difícil para el sur brasileño elevar al norte hasta su propio nivel como será para Italia del Norte rehabilitar el Mezzogiorno.» En otra parte es más explícito: «Podría decir que San Pablo ha arrebatado de manos europeas los beneficios de explotarlo. Así es como consideran los italianos del sur el poder económico de Milán y San Pablo es el Milán de Brasil. El contraste económico y la consecuente tensión psicológica entre el sur de Italia y Milán es familiar a los observadores europeos. Transfiérase Italia al hemisferio sur y magnifíquense sus dimensiones varias veces y se tendtá la relación de hoy en día entre el Norte de Brasil y San Pablo.»"

B) Lo mismo sucede a propósito de los países platenses, que Lambert pretende excluir de la dicotomía estructural societaria por haberse introducido en ellos la industrialización en fechas más tempranas. Para el Uruguay en 1956 nos corresponde afirmar: «Hay en el Uruguay dos sociedades superpuestas o, mejor, que coexisten en el mismo territorio. La primera es la sociedad moderna representada por Montevideo y las ciudades del interior e incluso por ciertas zonas rurales. En ellas predominan los caracteres de la sociedad urbana. [...] La otra sociedad -igualmente

9. Entre el Maule y el Amazonas (Santiago de Chile: Francisco de Aguirre, 1968), pp. 40 y 46. 
uruguaya - está unida a la gran propiedad agropecuaria con su sistema de trabajo secular de la Colonia.» ${ }^{10}$ En principio esto eta tanto como trazar la línea divisoria de las dos sociedades uruguayas incluyendo junto a la sociedad urbana buena parte de la zona agtatia (agricultura, hotticultura). Para Argentina, Gino Germani tetoma observaciones definitivas que Sarmiento había hecho anteriormente.

C) Los historiadores han destacado la necesidad de considerar el factor cronológico al establecer las situaciones dicotómicas. En otras palabras, podría encontrarse este tipo de cuadros referidos a una determinada época, pero aun en el mismo país los mismos no se aprecian pasado cierto tiempo. ${ }^{11}$

El tema reaparece en los trabajos sistemáticos de los actuales sociólogos profesionales con diferente lenguaje, pero tal vez sin trascender el alcance de los sociólogos del siglo pasado. En 1959 un seminatio en Santiago de Chile de UNESCO-CEPAL abordó desde diversos ángulos «La urbanización en América Latina», y aunque, desdichadamente, no se publicaton la totalidad de los trabajos monográficos presentados, ni tampoco los anales del seminario, un volumen recogió las ideas del coordinador, el profesor norteamericano Philip Hauser, de la Universidad de Chicago, que en principio ha reiterado ideas ya expresadas en obras antetiotes, a su vez coincidentes con planteos del profesor Gino Germani, en su obra Politica y sociedad en una época de transición (Buenos Aires: Paidós, 1962). De acuerdo a las mismas, sería posible elaborar una tipología «capaz de dar cuenta del proceso de urbanización desde la perspectiva sociológica. Para tal fin se concebían dos tipos de sociedades, una denominada sociedad tradicional y la otra sociedad industrial. Entre ambas se concebía una base intermedia que daba lugar a la Ilamada sociedad en tratrsición». ${ }^{12}$

En las sociedades tradicionales latinoamericanas uel tipo de acción predominante se encuentra fijado, prescrito; para cada situación de una manera más o menos rígida [...] desalienta el cambio y exalta más bien la herencia del pasado [y.] la mayoría de las funciones se concentran en unas pocas instituciones». La sociedad industrial (que Hauser asimila mecánicamente a la urbana), en cambio, tiene «el tipo de acción que cabe de-

10. Catlos M. Rama, Ensayo de sociología uruguaya (Montevideo: Medina, 1956, p. $301-302$.

11. Por ejemplo, Tulio Balperin Donghi, en su Historia contemporánea de América Latina (Madrid: Alianza Editorial, 1969), pp. 39 y ss., hace referencia al siglo xvm, pero para el mismo autor el cuadro es inaceptable en épocas posteriores.

12. Volumen La urbanización en América Latina (Buenos Aires: Solar-Hachette, 1967), pp. 48 y ss., correspondientes al informe Hauser. 
nominar deliberada [...] valora y estimula toda mudanza, es decir, el cambio se encuentra institucionalizado [y] dominan las instituciones especializadas, cada una con una tarea limitada y específica». El informe Hauser pone el acento especialmente en la importancia de las sociedades de transición, es decir, tecientes urbanizaciones visibles en varios de los países latinoamericanos, en que se mezclan elementos de la sociedad tradicional con la nueva industrial.

La historia y consideración del papel de las ciudades en América Latina no solamente es un tema central para la iniciación al análisis de la historia socio-conómica, sino que permite ampliarla sobre el problema de Ia dicotomía.

Richard M. Morse considera la existencia de dos grupos de interpretaciones: a saber, los difusionistas (para los cuales las ciudades son focos de los cuales se irradia la civilización a las campinas) y los que consideran a las ciudades centros de control, ya sea en beneficio de las oligarquías urbanas nacionales, ya como piezas de la dependencia exterior. ${ }^{13} \mathrm{El}$ mismo Morse introduce una nueva variante de la visión dicotómica, porque destaca que Estados Unidos ha sido estudiado a través de la confrontación de «los dos países» del algodón sureño y del trigo norteño, y que de la misma manera puede analizarse a países como Cuba (entre el oriente ganadero y el occidente azucarero), Colombia (dividido entre regiones latifundistas y de taller), y Argentina (litoral e interior).

Es obvio que, como en el caso de los Estados Unidos, hay un factor geográfico decisivo en varios de esos casos, y la diversidad de la producción no implica necesariamente la existencia de «dos países» tadicalmente diferenciados. Este planteo recuerda el del profesor Frédéric Mauro ya citado.

Razón tienen profesores como Faletto y Ruiz cuando apuntan que se trata de un nuevo lenguaje que insiste en el dualismo campo-ciudad que recogiera Marx de la economía política inglesa, y que -como hemos visto- en Argentina con Sarmiento, en Brasil con Lambert, en México con González Casanova, o en Uruguay con el autor ha sido explotada como hipótesis. ${ }^{14}$

13. Véase Las ciudades latinoamericanas (México: Sep-Setentas, 1973), vol. I, pp. 35 y ss., tema sobre el cual también abundará en La investigación urbana latinoamericana (Buenos Aires: SIAP, 1971).

14. Faletto y Ruiz, «Notas para una sociología de la urbanización en América Latina», Revista de Ciencias Sociales, núm. 2 (noviembre de 1971), pp. 11-28. Estos autores también señalan una presunta similitud entre la tipología de Hauser y la famosa de Töennies de comunidad y sociedad, entrando seguidamente al análisís de la situación chilena. 
Hay razones técnicas sin embargo para la insatisfacción. Son vatios los autores que apuntan que no toda la sociedad tural es tradicional, y posiblemente sería necesario afinar conceptos en la definición de sociedad urbana. Los censos demográficos Latinoamericanos usan criterios diferentes, y divergentes, para estimar la población urbana, y a estos efectos - a nuestro parecer- es básico tener en cuenta la participación de la mano de obra en los diferentes sectores socio-económicos.

Por otra parte lo que llama poderosamente la atención no es la mera distinción entre la ciudad y el campo, universal en todas las sociedades civilizadas y base de la división del trabajo, sino la distancia social entre dos tipos de sociedad, distancia que llega al antagonismo, y a un dualismo polarizado de tipo dicotómico. En la medida que en las ciudades se importa una tecnología reciente, especialmente para el observador europeo, la sociedad que contienen se muestra hasta como más moderna que la de sus respectivos países, mientras esto destaca el arcaísmo de los rurales.

Hay un uso del vocablo en sociología económica, pues, en la medida que una sociedad dicotómica supone una economía también dual, los economistas y sociólogos de la CEPAL han mejorado el concepto, ahora de dualismo económico.

Los dos sistemas económicos que conviven en América Latina, se caracterizarían asimismo por una distinta tecnología, y es explicable que en esos textos se haga referencia al dualismo tecnológico, e incluso al dualismo ecológico, por ejemplo, atendiendo al hecho de que las regiones costeras son más pobladas mientras el binterland es a menudo despoblado. Asimis* mo la dualidad económica, implicaría un dualismo en la distribución del ingreso, o se habla sobre «distribución del ingreso geográfico».

Estas conceptualizaciones, en el caso de economistas, se reclaman de las ideas de J. H. Boeke, en su obra Economies and policy of dual societies (Nueva York, 1953), destacando que nacidas para aplicarse a las sociedades africanas y asiáticas, es necesatio perfeccionar su uso en América Latina. «Las economías nacionales [con] sistemas duales [se caracterizan] por alta tecnología para la expansión fuera del área y deficiente tecrología para el mercado nacional», señala C. Matus. Hay una «heterogeneidad estructural», dice Aníbal Pinto. Estos autores consideran el concepto para explicar el «desarrollo interno», o «desde dentro», como contratio a la colonización o implantación foránea del desarrollo económico. ${ }^{\text {is }}$ Pero entre los economistas más recientes se anotan críticas a esta conceptuación.

Francisco Oliveira, del CEBRAP brasileño, en un reciente estudio

15. Véase el volumen Dos politicas sobre el desarrollo de América Latina (San- 
intitulado justamente «La economía brasileña: crítica a la razón dualista», ${ }^{16}$ se inscribe en una reacción al análisis cepalino dicotómico sociedad moder. na-sociedad tradicional que, a su parecer, ha sido una decisiva contribución de la economía para Ilevar «la sociología y la ciencia política a una especie de callejón sin salida rostowiano». A su parecer el concepto de subdesarrollo «como una formación histórico-económica particular, construida polarmente alrededor de la oposición formal de un sector atrasado y un sector moderno no se sostiene como originalidad. Este tipo de dualidad puede encontrarse no sólo en casi todos los sistemas, sino en casi todos los períodos. Por otra parte, la oposición en la mayoría de los casos es tan sólo formal. De hecho, el proceso real muestra una simbiosis y una organicidad, una unidad de conceptos opuestos, en la cual lo llamado moderno crece y se alimenta de la existencia de lo atrasado, si se quiere determinar la terminología». Y continúa Oliveira: «El subdesarrollo pa. recería la forma peculiar de ser de las economías preindustrializadas invadidas por el capitalismo y, poz tanto, en etapa transitoria hacia formas más avanzadas y sedimentadas de éste; sin embargo, tal planteamiento olvida que el subdesarrollo es precisamente un producto de la expansión del capitalismo [...] en resumen, el subdesarrollo es una formación capitalista, y no solamente históricas.»

En verdad hay cepalinos que refutan igualmente las tesituras dualistas, como Fernando Henrique Cardoso y Aníbal Reyna, en su trabajo Industrialización, estructura ocupacional y estratificación social en América Latina (México: Siglo XXI, 1972).

Dentro del marxismo se ha intentado el abordaje del tema, no tanto sobre la profundización de El Capital, sino considerando las hipótesis del desarrollo desigual, y hasta el antagonismo ciudad capitai-resto del país, muy importante en algunos países latinoamericanos. En este plano destaquemos, para aludir a un escritor contemporáneo, a los escritos de Ernesto Guevara tan ampliamente difundidos. ${ }^{17}$

La connotación política de la dicotomía estructural societaria latinoamericana no ba dejado de destacarse por todos los autores que han tratado el tema. Sarmiento basaba en ella la explicación de los partidos fede.

tiago de Chile: ILPES-CEPAL, 1970) con la intervención de Carlos Matus, Pedro Vuskovic, Aníbal Pinto y otros.

16. Francisco Oliveira, «La economía brasileña: crítica a la razón dualista», $E l$ Trimestre económico, vol. XL, núm. 158 (abril-junio de 1973), versión española de Adolfo Alarcón.

17. Ennesto Guevara, Condiciones para el desarrollo económico latinoamericano (Montevideo: EI Siglo Ilustrado, 1967), en que hemos recogido diversos textos del citado autor sobre asuntos económicos y sociales. 
ral (rural) y unitario (urbano), y su análisis lo confirmará medio siglo más tarde el primero de los sociólogos sistemáticos argentinos José Ingenieros, cuando considera que las guetras civiles de su país resultan de una rivalidad entre dos oligarquías pertenecientes a la misma clase social, pero con bases económicas antagónicas. ${ }^{18}$

Lambert, que llega a Brasil pocos años más tarde de la llamada «re. volución paulista) de 1932 (que enfrenta al Estado industrial de São Paulo contra la oligarquía terrateniente del resto del país), ve en ese proceso una reiteración de la Guerra de Secesión Norteamericana entre el industrializado norte y el latifundista y esclavista sur. Peto nuestro autor verá incluso cómo el programa industrializador y nacionalista de los paulistas será llevado adelante por la alianza política entre los industriales y los grandes latifundistas exportadores en la época de Getulio Vargas. Brasil, igual que Chile, examinado por Faletto y Ruiz (y podrían multiplicarse los ejemplos) demuestra que ta lucha política entre ambas oligarquías es - como decía Ingenieros - una mera disputa y puede ser obviada en un modelo político nacional de envergadura.

No hace mucho un dirigente militar de primera línea del régimen imperante en Brasil desde 1964 describia el plan de desarrollo social como favorable exclusivamente a 20 millones de brasileños, es decir, entonces el $25 \%$ de la población nacional. Éstos son los integrantes de las clases alta y media superior, y capas urbanas de técricos (los militares en primer término), coincidentes en su mayoría con el país nuevo de Lambert, pero excluyendo a la clase obrera, aunque incorporando al privilegio a la élite empresarial agraria.

La dicotomía sigue en pie, es un ejemplo a tener en cuenta en cualquier análisis social, económico y político, y una llave para la comprensión de la América Latina contemporánea. Seguramente resulta más útil en un contexto historicista, combinándola con el análisis de la estratificación social.

Carlos M. Rama

Departament de Sociologia

Universitat Autònoma de Barcelona

Bellaterra, Barcelona.

18. La evolución de las ideas en la Argentina (Buenos Aires: Bosso, 1937). Aco. temos que es distinta la opinión que el punto merece al fundador del Partido Socialista Argentino, doctor Juan B. Justo, inspirador a distancia del llamado «socialismo nacional is difundido en el Plata. 